\title{
Anisotropic Selection in Cellular Genetic Algorithms
}

\author{
David Simoncini \\ David.Simoncini@unice.fr \\ Sébastien Verel \\ Philippe Collard \\ Manuel Clergue \\ Sebastien.Verel@unice.fr \\ Philippe.Collard@unice.fr \\ Manuel.Clergue@unice.fr
}

I3S Laboratory, CNRS-Université de Nice Sophia Antipolis

\begin{abstract}
In this paper we introduce a new selection scheme in cellular genetic algorithms (cGAs). Anisotropic Selection (AS) promotes diversity and allows accurate control of the selective pressure. First we compare this new scheme with the classical rectangular grid shapes solution according to the selective pressure: we can obtain the same takeover time with the two techniques although the spreading of the best individual is different. We then give experimental results that show to what extent AS promotes the emergence of niches that support low coupling and high cohesion. Finally, using a cGA with anisotropic selection on a Quadratic Assignment Problem we show the existence of an anisotropic optimal value for which the best average performance is observed. Further work will focus on the selective pressure self-adjustment ability provided by this new selection scheme.
\end{abstract}

\section{Categories and Subject Descriptors}

I.2.8 [Problem Solving, Control Methods, and Search]: [Heuristic methods]

\section{General Terms}

Algorithms

\section{Keywords}

Evolutionary computation, combinatorial cptimization

\section{Introduction}

This paper deals with selective pressure and diversity in cellular genetic algorithms (cGAs) which are a subclass of Genetic Algorithms where the population is embedded in a grid. These concepts are closely related to the exploration/exploitation trade-off. Previous studies on cGAs selected the size and the shape of neighborhoods [13], or the shape of the grid $[2,5,6]$ as basic parameters to tune the

Permission to make digital or hard copies of all or part of this work for personal or classroom use is granted without fee provided that copies are not made or distributed for profit or commercial advantage and that copies bear this notice and the full citation on the first page. To copy otherwise, to republish, to post on servers or to redistribute to lists, requires prior specific permission and/or a fee.

GECCO'06, July 8-12, 2006, Seattle, Washington, USA.

Copyright 2006 ACM 1-59593-186-4/06/0007 ...\$5.00. search process. Altering these structural parameters entails a deep change in the way we deal with the problem. For instance, there is no built-in mean to swap from a rectangular grid to a square grid without misshaping the neighborhood relation. We suggest using anisotropic selection (AS) to promote diversity and to control accurately the selective pressure in genetic search. The main advantage of the anisotropic selection scheme is that it allows to control the exploration/exploitation trade-off without affecting neither the grid topology nor the neighborhood shape; so the cellular genetic algorithm we propose merely works on a square grid and a simple Von Neumann neighborhood shape.

The paper is divided in 6 sections. Section 1 gives a brief definition of cGAs and an overview of existing techniques used to control the exploration/exploitation tradeoff. Section 2 introduces the AS scheme. Section 3 studies the influence of AS on the selective pressure. In Section 4 we compare AS and rectangular grids topologies' influence on the selective pressure. In Section 5 we show how AS promotes the emergence of niches. In Section 6 we use a cGA on a Quadratic Assignment Problem to measure the correlation between anisotropy and performance. Finally we tie together the results of the previous sections and suggest directions for further research.

\section{SELECTION IN CELLULAR GENETIC ALGORITHMS}

This section presents a brief overview on cellular Genetic Algorithms and a standard technique to measure the selective pressure.

\subsection{Cellular Genetic Algorithms}

Cellular Genetic Algorithms are a subclass of Genetic Algorithms (GAs) in which exploration and population diversity are enhanced thanks to the existence of small overlapped neighborhoods [14]. Such algorithms are specially well suited for complex problems [9]. We assume a twodimensional toroidal grid as a spatial population structure. Each grid cell contains one individual of the population. The overlapping neighborhoods provide an implicit mechanism for migration of genetic material throughout the grid. A genetic algorithm is assumed to be running simultaneously on each grid cell, continuously selecting parents from the neighborhood of that grid cell in order to produce an offspring which replaces the current individual.

\subsection{Takeover Time}


A standard technique to study the induced selection pressure without introducing the perturbing effect of variation operators is to let selection be the only active operator, and then monitor the number of best individual copies $N(t)$ in the population [8]. The takeover time is the time it takes for the single best individual to conquer the whole population. The grid is initialized with one cell having the best fitness and all the other having a null fitness. Since no other evolution mechanism but selection takes place, we can observe the way the best individual spreads over the grid by counting generation after generation the number of copies of this one. A shorter takeover time thus means a higher selective pressure. It has been shown that when we move from a panmictic population, as in standard GA, to a spatially structured one of the same size with synchronous updating of the cells, the global selection pressure induced on the entire population is weaker [13].

Links have been established between takeover time and neighborhood size and shape or grid topology. Neighborhood size and shape in a cGA are parameters that have some influence on the takeover time. A larger overlap of local neighborhoods of the same shape speeds up the best individual's spreading over the grid. The influence of the shape is given by Sarma and De Jong through a measure on the neighborhood which represents the spatial dispersion of a cell pattern [13]. Rather than the size of the neighborhood in terms of individuals, the key particularity of a local neighborhood is its radius. The takeover time decreases while the radius increases in a spatially structured population.

We measure the relation between grid topology and selective pressure for rectangular grids where the population size is fixed to 4096 . We use the following grid shapes: $64 \times 64$; $32 \times 128 ; 16 \times 256 ; 8 \times 512 ; 4 \times 1024$ and $2 \times 2048$ individuals. The selection strategy is a binary tournament. For each cell we randomly choose two individuals in its neighborhood. The best one then replaces the individual of the cell on the grid if it is fitter or with probability 0.5 if fitnesses are equal. Figure 1 shows the average of $10^{3}$ independant runs of growth of $N(t)$ against generations; the takeover time is reached when $N(t)$ is equal to the size of the grid (see Table I). The average growth rate $\Delta(t)$, that is the number of new best individual copies per time unit, of these curves for four rectangular grid shapes $(64 \times 64,32 \times 128,16 \times 256,8 \times 512)$ is plotted in Figure 2] This figure helps us to understand the growth of $N(t)$. The growth rate $\Delta(t)$ is the same for all grids for the first generations. Then, the spreading speeds down to reach a constant speed for rectangular grids. This constant is $2 l p$ where $p$ is the probability of selecting the best individual when there is one copy of it in the neighborhood and $l$ the shortest side of the grid [5]. More accurately, $\Delta(t)$ decreases when $l$ is filled by copies of the best individual (see Figure 3(b)). Then, the speed becomes constant until the best individual has spread over to the other side (see Figure 3(c)). This explains why the $64 \times 64$ grid curve has no constant period: the two sides are filled at the same time. Finally, the growth rate falls down to zero as the best individual finishes conquering the grid. The results of the experiments we conducted are in agreement with E.Alba and J.Troya observations that narrow grid shapes induce low selective pressure [2]. We will see in the next sections that this behavior can be observed with the AS too.
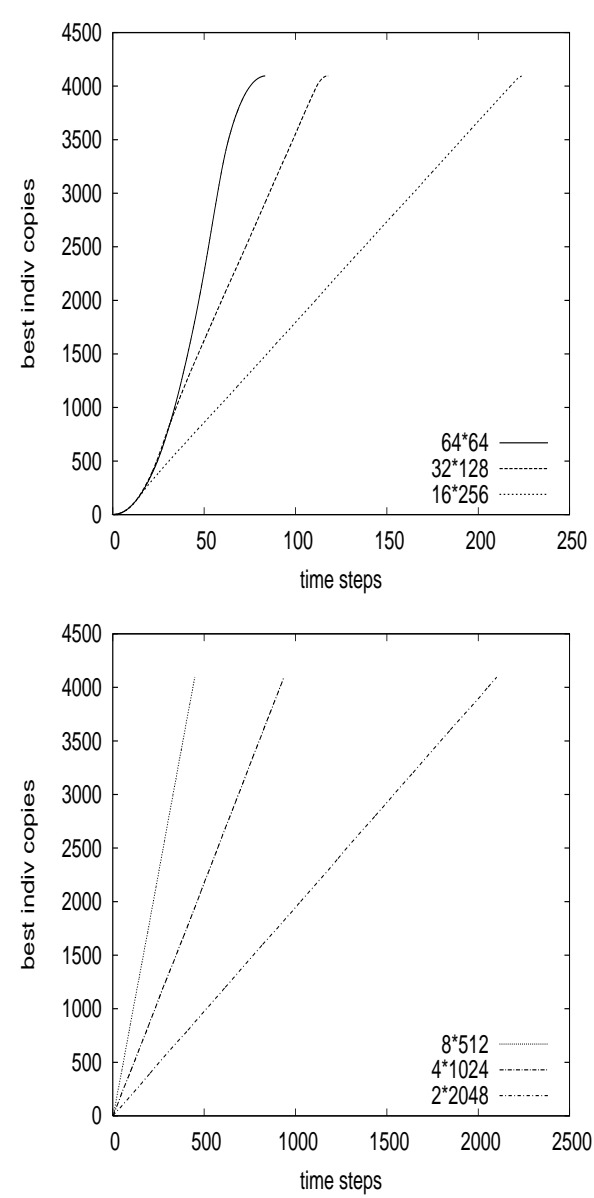

Figure 1: Growth curves of the number of best individual copies $N(t)$ on different grid shapes.

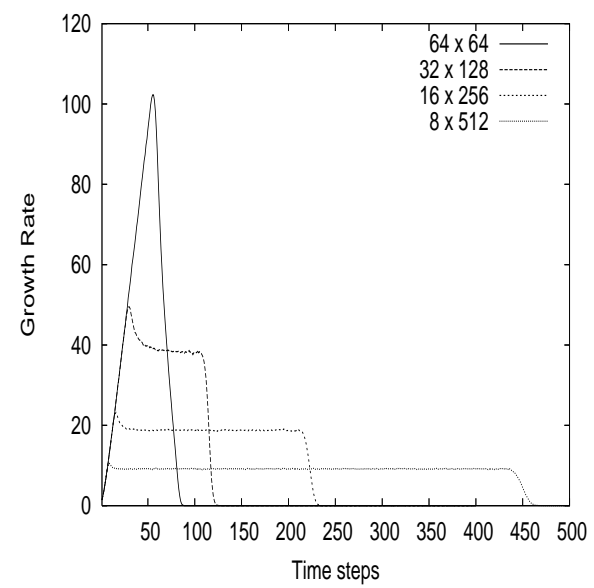

Figure 2: Growth rate against time steps for four rectangular grid shapes. 


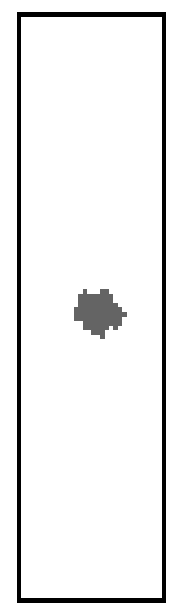

(a)

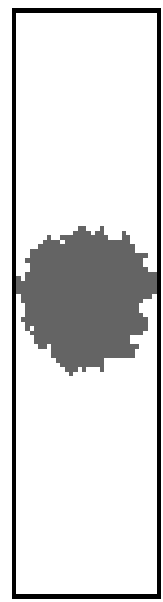

(b)

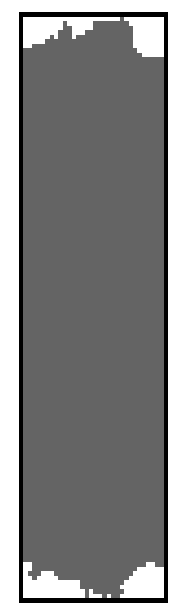

(c)
Figure 3: Spreading of the best individual over a $32 \times 128$ grid
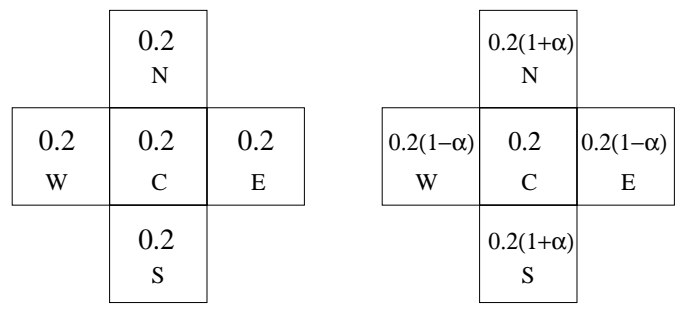

Figure 4: Von Neumann and Von Neumann fuzzy Neighborhoods with probabilities to choose each neighbor

Table 1: Takeover time for different rectangular grid shapes.

\begin{tabular}{|r|rrr|}
\hline & \multicolumn{3}{|c|}{ Takeover Time } \\
Grid shape & Avg & Min & Max \\
\hline $64 \times 64$ & $83.4_{1.9}$ & 79 & 87 \\
$32 \times 128$ & $117.8_{2.4}$ & 114 & 123 \\
$16 \times 256$ & $225.0_{3.8}$ & 219 & 232 \\
$8 \times 512$ & $449.7_{6.3}$ & 437 & 463 \\
$4 \times 1024$ & $937.1_{9.9}$ & 921 & 960 \\
$2 \times 2048$ & $2101.2_{29.9}$ & 2045 & 2155 \\
\hline
\end{tabular}

\section{ANISOTROPIC SELECTION}

This section introduces our contribution: the Anisotropic Selection method where the neighbors of a cell may be selected with different probabilities.

\subsection{Von Neumann Fuzzy Neighborhood}

The Von Neumann neighborhood of a cell $C$ is defined as the ball of radius 1 in Manhattan distance centered at $C$. Using the Von Neumann Fuzzy Neighborhood, we assign different probabilities to choose one cell in the neighborhood according to the directions (see Figure 4). The probability $p_{c}$ to choose the center cell $C$ is set at $\frac{1}{5}$ as for Von Neumann neighborhood. Let $p_{n s}$ denote the probability to choose the cell $N$ or $S$ and $p_{\text {ew }}$ denote the probability to choose the cell $E$ or $W$. Let $\alpha \in[-1 ; 1]$ be the control parameter, the anisotropy degree. When $\alpha=-1$, we have $p_{\text {ew }}=1-p_{c}$ and $p_{n s}=0$, when $\alpha=0$ we have $p_{n s}=p_{\text {ew }}$ and when $\alpha=1$ we have $p_{n s}=1-p_{c}$ and $p_{e w}=0$. Thus, the probabilities $p_{n s}$ and $p_{\text {ew }}$ can be described as:

$$
\begin{aligned}
& p_{\text {ns }}=\frac{\left(1-p_{c}\right)}{2}(1+\alpha) \\
& p_{\text {ew }}=\frac{\left(1-p_{c}\right)}{2}(1-\alpha)
\end{aligned}
$$

The case $\alpha=0$ correspond to the standard Von Neumann neighborhood $\left(p_{n s}=p_{\text {ew }}=\frac{2}{5}\right)$ and $\alpha=1$ is the limiting case for fuzzy neighborhood where $p_{n s}=\frac{4}{5}$ and $p_{\text {ew }}=0$. In the latter case, there is a vertical neighborhood with three neighbors only 1 .

\subsection{Definition}

The AS operator exploits the Von Neumann Fuzzy Neighborhood. It works as follows: for each cell $k$ individuals are selected accordingly to the probabilities $p_{n s}, p_{\text {ew }}$ and $p_{c}(k$ stands in the range $[1,5])$ within the cell neighborhood. The $k$ individuals participate to a tournament and the winner replaces the old individual if it is fitter or with probability 0.5 if fitnesses are equal. The control parameter $\alpha$ is a measure of anisotropy: $\alpha=0$ corresponds to standard selection, and $\alpha=1$ is the limiting case with the utmost anisotropy. We conjecture that selective pressure decreases when anisotropy increases.

\section{SELECTIVE PRESSURE AND ANISOTROPIC SELECTION}

In this section we study the relationship between selective pressure and AS. First, we measure the takeover time for different anisotropy degrees, then we take the study further by considering the growth curve of the best individual.

We measure the effect of different anisotropy degrees on the takeover time. In our experiments, the anisotropic selection is based on tournament selection of size $k=2$ on the square grid of side 64 . All the 4096 cells are updated synchronously. For each cell, we substitute the selected individual to the one already present in that cell, either systematically if the selected individual is fitter, or with probability 0.5 if fitnesses are equal. For each value of $\alpha$, we perform $10^{3}$ independent runs. When $\alpha=0$, no direction is privileged and $\mathrm{AS}$ is equivalent to the standard selection method. When $\alpha=1$, only one direction is exploited, the grid can not be filled and the takeover time is not defined. Figure 5 shows the influence of AS on the takeover time : it increases with the parameter $\alpha$. These results are fairly consistent with our expectation that selection intensity decreases when the anisotropic degree increases. However, the correlation between takeover and anisotropy is not linear; it rapidly increases after the value $\alpha=0.9$.

\footnotetext{
${ }^{1}$ using the grid symmetry we will consider $\alpha \in[0 ; 1]$ only
} 


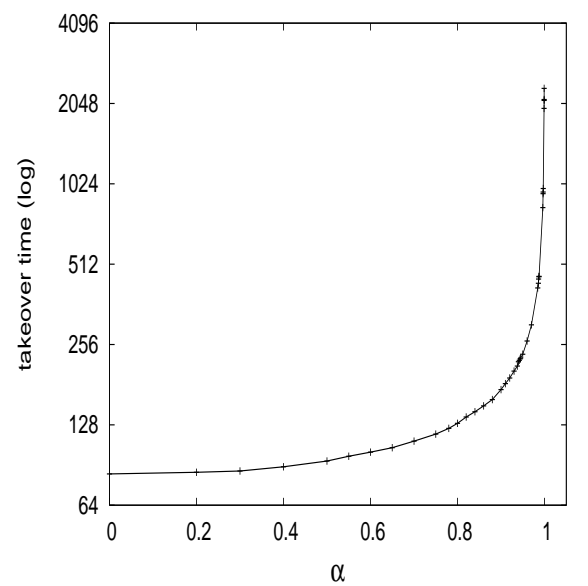

Figure 5: Average of the takeover time as a function of the anisotropic degree $\alpha$.

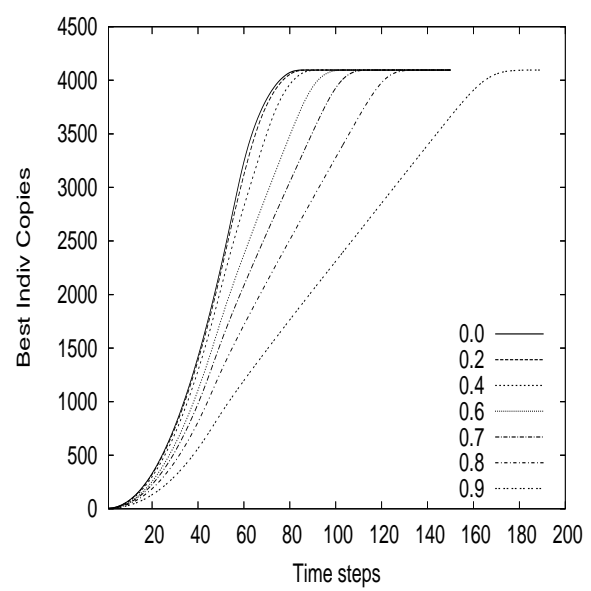

(a)

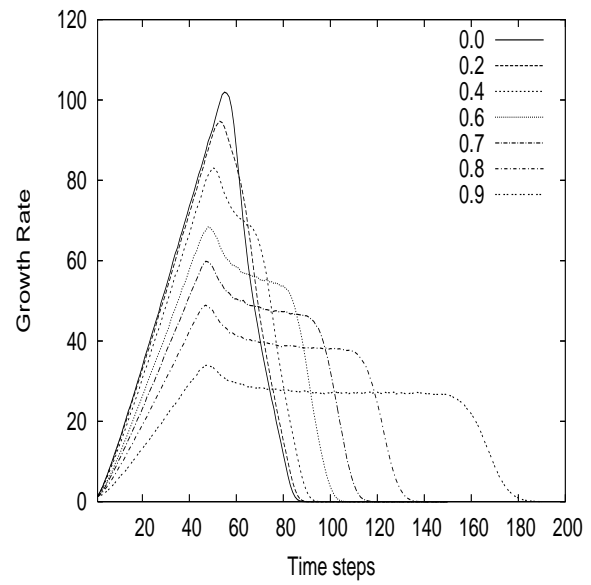

(b)

Figure 6: Growth curves of $N(t)$ (a) and $\Delta(t)$ (b) on a square grid for different anisotropic degrees $\alpha$.

Figure 6] shows the average curves of the growth of best
Table 2: Values of $\alpha$ and $\frac{l}{L}$ for the same takeover time. Linear regression shows the relation between $\alpha$ and $\frac{l}{L}$ by the equation $\alpha=-0.999 \frac{l}{L}+0.998$ with high correlation coefficient -0.9999

\begin{tabular}{|l|l|l|}
\hline \multicolumn{1}{|c|}{$l / L$} & \multicolumn{1}{c|}{$\alpha$} & takeover time \\
\hline 0.000977 & 0.99911 & 2101 \\
0.003906 & 0.99674 & 939 \\
0.015625 & 0.9864 & 450 \\
0.0625 & 0.944 & 225 \\
0.25 & 0.75 & 118 \\
1.0 & 0.0 & 83 \\
\hline
\end{tabular}

individual (a) and its growth rate (b) as a function of time steps. The shape of the curve is decomposed into three stages: in the first stage, the growth rate is almost proportional to the time steps and the growth curve is approximatively a parabola. In the second stage, this rate becomes roughly constant after a period of decrease and the growth curve is almost linear. In the last stage, the rate decreases linearly down to zero with a different slope than in the first stage. The higher the anisotropic degree $\alpha$, the weaker the initial slope of the growth rate. In the same way in the second stage, the slope of growth curve is smaller when $\alpha$ is higher. Therefore, the selective pressure is lower when anisotropy is higher.

The three stages of the growth curve correspond to three periods in the spreading of the best individual on the square grid (see Figure 7). During the first stage, the best individual spreads more in the privileged direction. This period finishes, as described in Section [1.2, when a side of the grid is filled by best individual copies in the privileged direction (see Figure [7(b)). During the second period, the best individual fills the second direction of the grid until it has spread over a side of it in the less privileged direction (see Figure 77(c)). The best individual front is sharp at the beginning, and becomes approximatively a horizontal line later. The third time finishes to fill the grid. Taking into account these three phases, one may be able to give the equation of the growth curve as in [5].

\section{ANISOTROPIC SELECTION VS. RECTANGULAR GRID}

Changing rectangular grid shape and tuning the anisotropic degree are two methods for varying the selective pressure. This section compares the two methods and shows in which way they are equivalent.

From the experimental results presented in Sections 1.2 and 3 we compute the $\alpha$ parameter value for which we obtained the same takeover time as for one particular rectangular grid shape. In Table II we give the $\alpha$ and $\frac{l}{L}$ values and exhibit the correlation between these parameters. We can see that $\alpha$ is proportional to the $\frac{l}{L}$ ratio. So, according to the takeover time using $\alpha=1-\frac{l}{L}$, it is possible to have the same selective pressure using the two methods.

Figure 8 shows the mean growth curves of the best individual spreading against time steps for all grid shapes and for the corresponding square grids using AS. Although, using the relation $\alpha=1-\frac{l}{L}$, we found the same takeover time with rectangular grid shapes and with AS the selective pressure is applied in a different way for the two methods. 


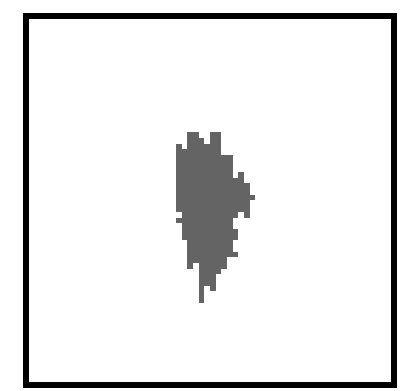

(a)

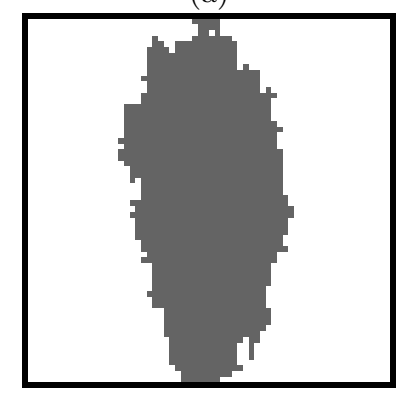

(b)

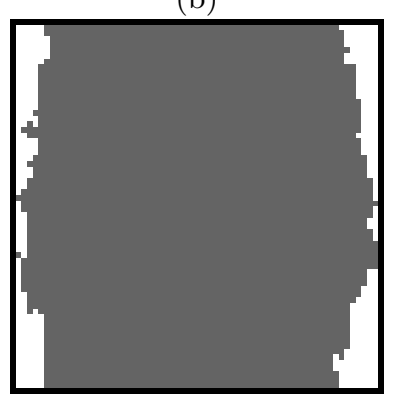

(c)

Figure 7: Spreading of the best individual with $\alpha=0.75$

It is weaker during the first generations in the anisotropic case then it becomes slightly stronger, to finally fill the grid at the same takeover time.

\section{ANISOTROPIC SELECTION AND NICHING}

Many real optimization problems require the coexistence of diverse solutions during the search. In this section we show how the anisotropic selection is able to promote niching.

\subsection{Niching methods}

Niching methods have been proposed in the field of genetic algorithms to preserve population diversity and to allow the GA to investigate many peaks in parallel. As a side effect, niching prevents the GA from being trapped in local optima. Niching methods are inspired from nature where species specialize themselves to different ecological niches in order to decrease the selective pressure they undergo. Niching GA's tend to achieve a natural emergence of niches in the search space. A niche is commonly referred to as an optimum of the domain, the fitness representing the resources of that
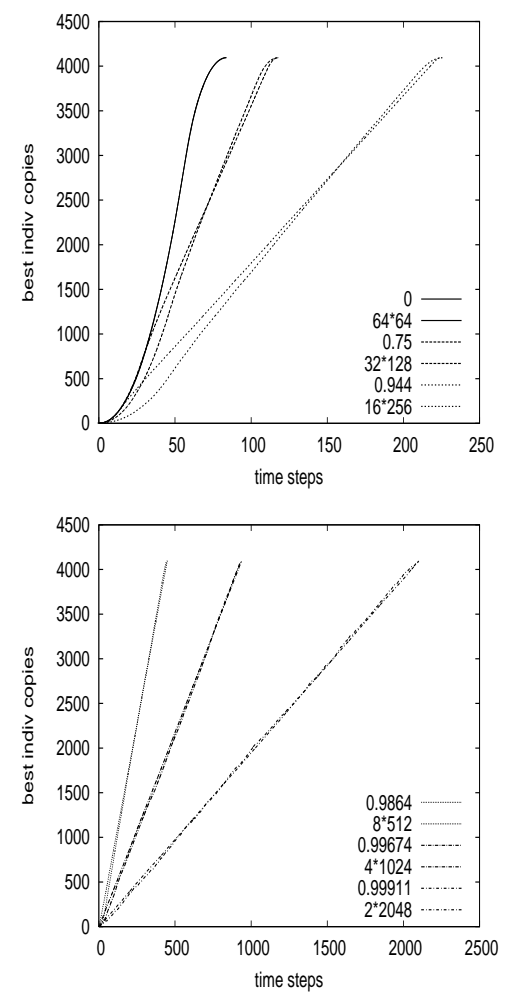

Figure 8: Comparison between growth curves on different rectangular grids and on a square grid with the equivalent anisotropy degree.

niche [12]. Niching methods are used to solve multimodal problems, and also in dynamic optimization [4]. For such problems a GA must maintain a diverse population that can adapt to the changing landscape and locate better solutions dynamically. There are different niching GA for panmictic population: sharing, crowding, etc. These methods are based on the concept of distance: sharing [7] decreases the fitness according to the number of similar individuals in the population, and with crowding, replacement is performed considering the distance between solutions.

\subsection{Experimental results}

To show up to what extent anisotropic selection promotes niching, we have conducted experiments where two solutions with the best fitness (here 1) are placed on a $64 \times 64$ square grid at the initial generation. These solutions are farther from each other in the least favored direction (here oriented horizontally). Figure 5.2 shows some snapshots of the spreading of the two bests over generations for different anisotropic degrees. Cells in light grey (resp. dark grey) are copies of the first best (resp. the second best), and all white cells have a null fitness value. Generations grow from top to bottom, and the anisotropic parameter $\alpha$ increases from left to right. The left-hand row $(\alpha=0)$ represents standard binary tournament schema ; we observe that standard selection is not able to maintain niches, after 1000 generations the grid is a mixture of the two optima. On the other hand, as $\alpha$ increases, two stable frontiers between niches emerge. Hence AS increases cohesion in each cells lineages. 


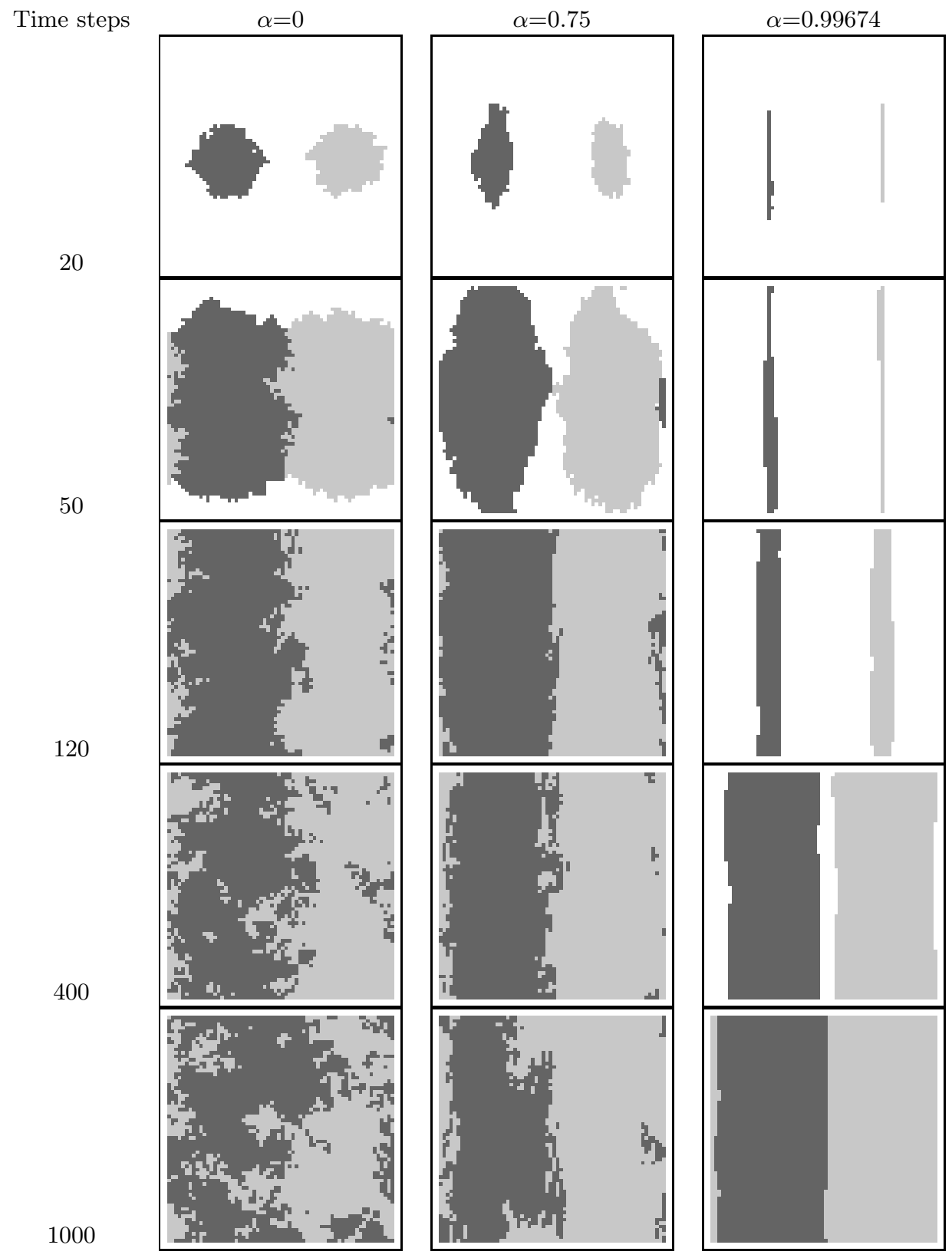

Figure 9: Spreading of two copies of the best individual 


\section{TEST PROBLEM}

We experiment a cGA using anisotropic selection on a Quadratic Assignment Problem (QAP): Nug30. Our aim here is not to obtain better results with respect to other optimization methods, but rather to observe the behavior of a cGA with AS. In particular, we seek an optimal value for the anisotropy degree.

\subsection{The Quadratic Assigment Problem}

The QAP is an important problem in both theory and practice. It was introduced by Koopmans and Beckmann in 1957 and is a modal for many practical problems [10].

The QAP can be described as the problem of assigning a set of facilities to a set of locations with given distances between the locations and given flows between the facilities. The goal is to place the facilities on locations in such a way that the sum of the products between flows and distances is minimal.

Given $n$ facilities and $n$ locations, two $n \times n$ matrices $D=$ $\left[d_{k l}\right]$ and $F=\left[f_{i j}\right]$ where $d_{k l}$ is the distance between locations $k$ and $l$ and $f_{i j}$ the flow between facilities $i$ and $j$, the objective function is:

$$
\Phi=\sum_{i} \sum_{j} d_{p(i) p(j)} f_{i j}
$$

where $p(i)$ gives the location of facility $i$ in the current permutation $p$.

Nugent, Vollman and Ruml suggested a set of problem instances of different sizes noted for their difficulty [3]. The instances they suggested are known to have multiple local optima, so they are difficult for a genetic algortihm. We experiment our algorithm on their 30 variables instance called Nug30.

\subsection{Experiments}

We consider a population of 400 individuals placed on a square grid. Each individual represents a permutation of $\{1,2, \ldots, 30\}$. We need a special crossover that preserves the permutations:

- Select two individuals $p_{1}$ and $p_{2}$ as genitors.

- Choose a random position $i$.

- Find $j$ and $k$ so that $p_{1}(i)=p_{2}(j)$ and $p_{2}(i)=p_{1}(k)$.

- swap positions $i$ and $j$ from $p_{1}$ and positions $i$ and $k$ from $p_{2}$.

- repeat $n / 3$ times this procedure where $n$ is the length of an individual.

This crossover is an extended version of the UPMX crossover proposed in [11]. The mutation operator consists in randomly selecting two positions from the individual and exchanging these positions. The crossover rate is 1 and we perform one mutation per individual in average.

We consider 500 runs for each anisotropy degree. Each run stops after 1500 generations.

Figure 10 shows the average performance of the algorithm towards $\alpha$ : for each value of $\alpha$ we average the best solution of each run. Performances are growing with $\alpha$ and then fall down as $\alpha$ is getting closer to its limit value. This curve shows the influence of the selective pressure on the performances and how important it is to control it accurately.
The best average performance is observed for $\alpha=0.86$, which corresponds to a good exploration/exploitation tradeoff. In the neighborhood of this optimal value the algorithm favors propagation of good solutions in the vertical direction with few interactions on the left or right sides. This kind of dynamics is well adapted to multi-modal problems as we can reach local optima on each columns of the grid and then migrate them horizontally to find new solutions.

Performances would probably improve if the selective pressure did not remain static during the search process. As in [1], we can define some criteria to self-adjust the anisotropy degree along generations. Furthermore, we can assign a different anisotropy degree to each cell of the grid, so that we can determinate criteria to self-adjust selective pressure locally upon measures on neighborhoods.

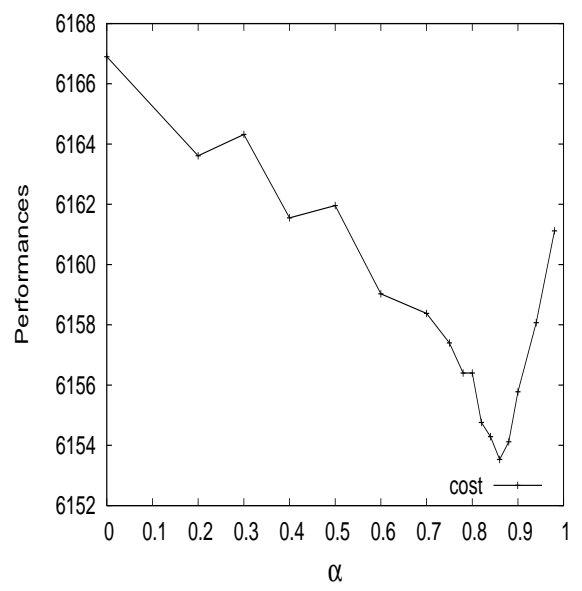

Figure 10: Average costs as a function of $\alpha$.

\section{Conclusions and Perspectives}

This paper presents a new selection scheme in cellular genetic algorithms. The main objective is to control the exploration/exploitation tradeoff in a flexible way. We propose to exploit the cellular GA characteristics to promote diversity during a genetic search process. Previous studies on cGAs selected structural parameters, as neighborhood or grid shape, to tune the selective pressure. The main drawback of these techniques is that altering a structural parameter entails a deep change in the way we deal with the problem. The new selection scheme we suggest is based on fuzzy neighborhood where a cell is chosen according to different probabilities. In order to favor one direction rather than the other one, anisotropic selection chooses individuals in fuzzy neighborhood. Experiments performed in order to establish relation between the takeover time and the degree of anisotropy are consistent with our expectation that selection pressure decreases with the degree of anisotropy. Analysis of the growth curves allows to distinguish three different phases in the diffusion process. Experimental results establish linear correlation in takeover between AS and cGA using rectangular grid. Then we point out capabilities of AS to promote the emergence of niches. Finally, using a cGA with AS on a QAP we have shown the existence of an anisotropic optimal value of $\alpha$ such that the best average performance is observed. 
This paper is a preliminary investigation and a more extensive analysis must be made to confirm that equilibrium between exploration and exploitation makes AS a good technique for complex problems in static or dynamic environments. Future work should address the following issues: comparison between AS and changes in the neighborhood shape and size, measuring AS effects with cGA using mutation and crossover, change the balance of directions dynamically. The latter point is an important feature: by tuning the control parameter $\alpha$, it would be possible to make the algorithm to self-adjust the selective pressure, depending on global or local measures. This adaptive ability has two important advantages: first, parameter $\alpha$ may vary in a continuous way, second, variations of this parameter have affect neither on the grid topology nor on the neighborhood shape. Such self-adaptive algorithms have been studied in previous works, but they need to change the grid topology to control the selective pressure [1], which means it is uniform in the grid. AS allows different propagation speeds on each area of the grid, promoting diversification and intensification (exploitation) at the same time on different spots. In general, we have to continue investigation of Anisotropic Selection to assess its validity and generality.

\section{REFERENCES}

[1] E. Alba and B. Dorronsoro. The exploration/exploitation tradeoff in dynamic cellular genetic algorithms. In IEEE transactions on Evolutionnary Computation, volume 9, pages 126-142, 2005.

[2] E. Alba and J. M. Troya. Cellular evolutionary algorithms: Evaluating the influence of ratio. In PPSN, pages 29-38, 2000.

[3] J. R. C.E. Nugent, T.E. Vollman. An experimental comparison of techniques for the assignment of techniques to locations. Operations Research, 16:150-173, 1968.
[4] W. Cedeño and V. R. Vemuri. On the use of niching for dynamic landscapes. IEEE International Conference on Evolutionary Computation, pages 1-6, 1997.

[5] M. Giacobini, E. Alba, A. Tettamanzi, and M. Tomassini. Modeling selection intensity for toroidal cellular evolutionary algorithms. In GECCO, pages 3-11, 2004.

[6] M. Giacobini, A. Tettamanzi, and M. Tomassini. Modelling selection intensity for linear cellular evolutionary algorithms. In P. L. et al., editor, Artificial Evolution, Sixth International Conference, Evolution Artificielle, EA 2003, Lecture Notes in Computer Science, pages 345-356, Marseille, France, October 2003. Springer.

[7] D. E. Golberg and J. Richardson. Genetic algorithms with sharing for multimodal function optimization. International Conference on Genetic Algorithms, pages 41-49, 1987.

[8] D. E. Goldberg and K. Deb. A comparative analysis of selection schemes used in genetic algorithms. In FOGA, pages 69-93, 1990.

[9] K. A. D. Jong and J. Sarma. On decentralizing selection algorithms. In ICGA, pages 17-23, 1995.

[10] T. Koopmans and M. Beckmann. Assignment problems and the location of economic activities. Econometrica, 25(1):53-76, 1957.

[11] V. V. Migkikh, A. P. Topchy, V. M. Kureichik, and A. Y. Tetelbaum. Combined genetic and local search algorithm for the quadratic assignment problem.

[12] B. Sareni and L. Krähenbühl. Fitness sharing and niching methods revisited. IEEE transactions on evolutionary computation, 2(3):1-9, 1998.

[13] J. Sarma and K. A. De Jong. An analysis of the effects of neighborhood size and shape on local selection algorithms. In PPSN, pages 236-244, 1996.

[14] P. Spiessens and B. Manderick. A massively parallel genetic algorithm: Implementation and first analysis. In $I C G A$, pages 279-287, 1991. 